\title{
The Data-Flow Equations of Checkpointing in Reverse Automatic Differentiation
}

\author{
Benjamin Dauvergne and Laurent Hascoët \\ INRIA Sophia-Antipolis, TROPICS team, \\ 2004 Route des lucioles, BP 93, 06902 Sophia-Antipolis, France
}

\begin{abstract}
Checkpointing is a technique to reduce the memory consumption of adjoint programs produced by reverse Automatic Differentiation. However, checkpointing also uses a non-negligible memory space for the so-called "snapshots". We analyze the data-flow of checkpointing, yielding a precise characterization of all possible memory-optimal options for snapshots. This characterization is formally derived from the structure of checkpoints and from classical data-flow equations. In particular, we select two very different options and study their behavior on a number of real codes. Although no option is uniformly better, the so-called "lazy-snapshot" option appears preferable in general.
\end{abstract}

\section{Introduction}

Mathematical derivatives are a key ingredient in Scientific Computation. In particular, gradients are essential in optimization and inverse problems. The methods to compute gradients can be classified in two categories. In the first category, methods use CPU more intensively because several operations are duplicated. This can be through repeated tangent directional derivatives, or through reverse Automatic Differentation using the "Recompute-All" strategy. This is not the context of this paper. In the second category, methods spare duplicated operations through increased memory use. This encompasses hand-coded resolution of the "adjoint equations" and reverse Automatic Differentation using the "StoreAll" strategy, which is the context of this work.

Being a software transformation technique, reverse AD can and must take advantage from software analysis and compiler technology [1] to minimize these efficiency problems. In this paper, we will analyze "checkpointing", an AD technique to trade repeated computation for memory consumption, with the tools of compiler data-flow analysis. Checkpointing offers a range of options that influence the resulting differentiated code. Our goal is to formalize these options and to find which ones are optimal. This study is part of a general effort to formalize all the compiler techniques useful to reverse $\mathrm{AD}$, so that $\mathrm{AD}$ tools can make the right choices using a firmly established basis.

\section{Reverse Automatic Differentiation}

Automatic Differentiation by program transformation takes a program $P$ that computes a differentiable function $F$, and creates a new program that com- 
putes derivatives of $F$. Based on the chain rule of calculus, AD iserts into $P$ new "derivative" statements, each one corresponding to one original statement of $P$. In particular, reverse $\mathrm{AD}$ creates a program $\bar{P}$ that computes gradients. In $\bar{P}$, the derivative statements corresponding to the original statements are executed in reverse order compared to $P$. The derivative statements use some of the values used by their original statement, and therefore the original statements must be executed in a preliminary "forward sweep" $\vec{P}$, which produces the original values that are used by the derivative statements forming the "backward sweep" $\overleftarrow{P}$. This is illustrated by Fig. 1, in which we have readily split $P$ in three successive parts, $U$ Upstream, $C$ Center, and $D$ Downstream. In our context, original values are made available to the backward sweep through PUSH and POP routines, using a stack that we call the "tape". Not all original values

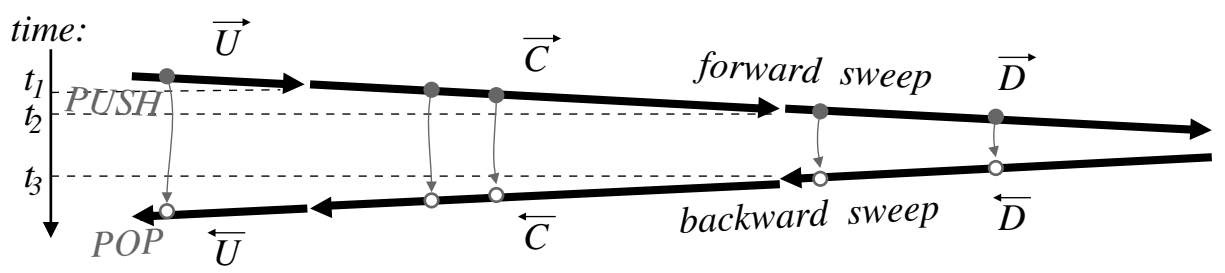

Fig. 1. Basic structure of reverse differentiated programs

are required in the backward sweep. Because of the nature of differentiation, values that are used only "linearly" are not required. The "To Be Recorded" (TBR) analysis [2, 6] finds the set of these required values denoted by Req. Set Req evolves as the forward sweep advances. For example in Fig. 1 TBR analysis of $U$ finds the variable values required by $\overleftarrow{U}$ (i.e. actually use $(\overleftarrow{U})$ ) which must be preserved between the end of $\vec{U}$ and the beginning of $\overleftarrow{U}$. To this end, each time a required value is going to be overwritten by a statement, it is PUSH'ed beforehand, and it is POP'ped just before the derivative of this statement.

Although somewhat complex, reverse AD can be easily applied by an automatic tool, and has enormous advantages regarding the number of computation steps needed to obtain the gradient [4, chapter 3].

In [5], we studied the data-flow properties of reverse differentiated programs, in the basic case of Fig. 1 i.e. with no checkpointing. We formalized the structure of these programs and derived specialized data-flow equations for the "adjoint liveness" analysis, which finds original statements that are useless in the differentiated program, and for the TBR analysis. In this paper, we will focus on the consequences of introducing checkpointing. In this respect this paper, although stand-alone, is a continuation of [5]. 


\section{The Equations of Checkpointing Snapshots}

Checkpointing modifies the differentiated code structure to reduce the peak memory consumption. When code fragment $C$ is "checkpointed" (notation $[C]$ ), the adjoint now written $\overline{[C] ; D}$ is formally defined by the recursive rewrite rule:

$$
\begin{aligned}
& R e q \vdash \overline{[C] ; D}=\operatorname{PUSH}(S b k) ; \\
& \text { PUSH (Snp); } \\
& C \text {; } \\
& R e q_{D} \vdash \bar{D} \\
& \text { POP }(\text { Snp }) \text {; } \\
& \begin{array}{l}
R e q_{C} \vdash \bar{C} \\
\text { POP }(S b k) ;
\end{array}
\end{aligned}
$$

Boxes show terms to be rewritten, whereas terms outside boxes are plain pieces of code. This new code structure is sketched in Fig, 2, to be compared with Fig. 1. Now, $C$ is first run in its original version, so that the tape consumed by

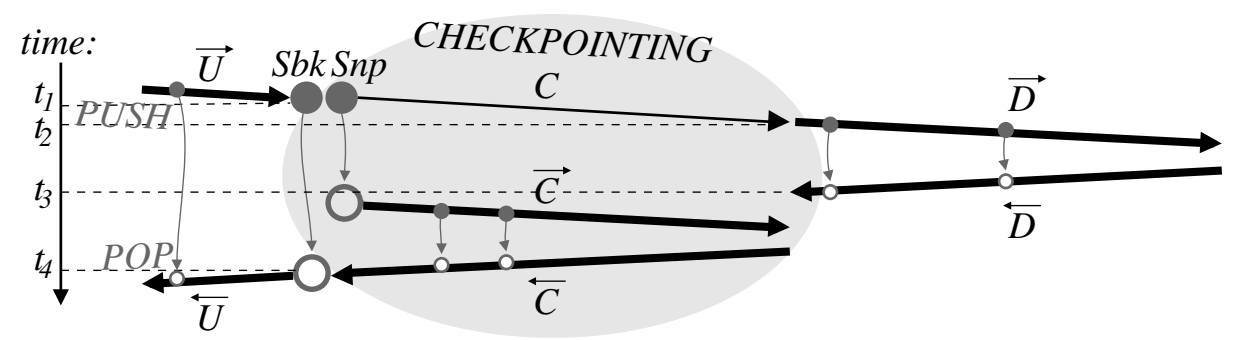

Fig. 2. Checkpointing in reverse $\mathrm{AD}$

$\bar{D} \doteqdot \vec{D} ; \overleftarrow{D}$ ("“; denotes code sequence) is freed before execution of $\bar{C} \doteqdot \vec{C} ; \overleftarrow{C}$ The peak memory consumption for $\overline{[C] ; D}$ is thus reduced to the maximum of the peak after $\vec{C}$ and the peak after $\vec{D}$. However, duplicate execution of $C$ requires that "enough" variables (the "snapshot" $S n p$ ) are preserved to restore the context of execution. This also uses memory space, although less than the tape for $\vec{C}$. To not lose the benefit of checkpointing, it is therefore essential we find the smallest snapshot for a fixed $C$, and in further studies the placement of $C$ that uses least memory.

This proves tricky: a larger snapshot can mean smaller tapes, and conversely. Therefore, unlike what happens with no checkpoints, there is no unique best choice for these sets. There are several "optimal" choices, among which none is better nor worse than the others. Our goal is to establish the constraints that define and link the "snapshot" and "tape" sets, and to characterize all the optimal choices. For our AD tool TAPENADE, we settled on one solution ( $c f$ Sect. (4) that our benchmarks indicated as a mean best choice. 
Four Unknown Sets of Variables. Let's examine checkpointing definition (1) in more detail. The rewrite context Req is the incoming "required set" of variables imposed by $U$, that must be preserved across execution of $R e q \vdash \overline{[C] ; D}$. On the other hand, $R e q_{D}$ and $R e q_{C}$ are the sets of variables that $\vdash \bar{C}$ and $\vdash \bar{D}$ will be required to preserve, respectively. For us, $R e q_{D}$ and $R e q_{C}$ are unknowns, to be determined together with the snapshot. About the snapshot itself, due to the stack structure, there are two places where variables may be restored from the stack: before $\vec{C}$ and before $\overleftarrow{U}$. Therefore we introduce two snapshot sets: Snp, the "usual snapshot", contains variables to be restored just before $\bar{C}$, thus ensuring that their value is the same for both executions of $C$. Sbk, the "backward snapshot", contains variables to be restored just before $\overleftarrow{U}$. Thus, whatever happens to these variables during $R e q \vdash \overline{[C] ; D}$, their value is preserved for $\overleftarrow{U}$. Using $S b k$ instead of $S n p$ and $R e q_{C}$ may improve memory traffic. In total, we have four "unknown" sets to choose: $R e q_{D}, R e q_{C}, S b k$ and $S n p$. Those sets must respect constraints parameterized upon $R e q, R e q_{D}, R e q_{C}, S b k, S n p$, and upon the fixed data-flow sets use (variables used) and out (variables partly written) of the code fragments $C, D, \bar{C}$, and $\bar{D}$. These constraints will guarantee that checkpointing preserves the semantics, i.e. the computed derivatives.

Two Necessary and Sufficient Conditions. Fig. 1] shows the differentiated program in the reference case with no checkpointing. This reference program is assumed correct. All we need to guarantee is that the result of the differentiated program, i.e. the derivatives, remain the same when checkpointing is done. This can be easily formulated in terms of data-flow sets. We observe that the order of the backward sweeps is not modified by checkpointing. Therefore the derivatives are preserved if and only if the original, non-differentiated variables that are used during the backward sweeps hold the same values. In other words, the snapshot and the tape must preserve the use set of $\bar{C}$ between time $t_{1}$ and $t_{3}$ i.e.

$$
\text { out }\left(\begin{array}{l}
\operatorname{PUSH}(S b k) ; \\
\operatorname{PUSH}(S n p) ; \\
C ; \\
R e q_{D} \vdash \bar{D} ; \\
\operatorname{POP}(S n p) ;
\end{array}\right) \bigcap \mathbf{u s e}\left(R e q_{C} \vdash \bar{C}\right)=\emptyset
$$

and the use set of $\overleftarrow{U}$, which is $R e q$ by definition, between time $t_{1}$ and $t_{4}$ i.e.

$$
\text { out }\left(\begin{array}{l}
\operatorname{PUSH}(S b k) ; \\
\operatorname{PUSH}(S n p) ; \\
C ; \\
R e q_{D} \vdash \bar{D} ; \\
\operatorname{POP}(S n p) ; \\
R e q_{C} \vdash \bar{C} ; \\
\operatorname{POP}(S b k) ;
\end{array}\right) \bigcap \operatorname{Req}=\emptyset \text {. }
$$

The rest is purely mechanical. Classically, the out set of a code sequence is:

$$
\operatorname{out}(A ; B)=\operatorname{out}(A) \cup \operatorname{out}(B),
$$


except in the special case of a PUSH/POP pair, which restore their argument:

$$
\operatorname{out}(\operatorname{PUSH}(v) ; A ; \operatorname{POP}(v))=\operatorname{out}(A) \backslash\{v\} .
$$

Also, the mechanism of reverse AD ensures that the variables in the required context are actually preserved, and this does not affect the variables used. Writing for short $\bar{A} \doteqdot \emptyset \vdash \bar{A}$, we have:

$$
\begin{aligned}
& \operatorname{out}(\operatorname{Req} \vdash \bar{A})=\operatorname{out}(\bar{A}) \backslash \operatorname{Req} \\
& \operatorname{use}(\operatorname{Req} \vdash \bar{A})=\operatorname{use}(\bar{A}) .
\end{aligned}
$$

Also, a PUSH alone overwrites no variable. Therefore, equation (2) becomes:

$$
\left(\operatorname{out}(C) \cup\left(\operatorname{out}(\bar{D}) \backslash R e q_{D}\right)\right) \backslash \operatorname{Snp} \bigcap \mathbf{u s e}(\bar{C})=\emptyset
$$

and equation (3) becomes:

$$
\left(\left(\operatorname{out}(C) \cup\left(\operatorname{out}(\bar{D}) \backslash R e q_{D}\right)\right) \backslash S n p \cup\left(\operatorname{out}(\bar{C}) \backslash R e q_{C}\right)\right) \backslash S b k \bigcap \operatorname{Req}=\emptyset .
$$

From (4) and (5), we obtain equivalent conditions on $S b k$, Snp, $R e q_{D}$ and $R e q_{C}$ :

$$
\begin{aligned}
& S b k \supseteq\left(\left(\operatorname{out}(C) \cup\left(\operatorname{out}(\bar{D}) \backslash \operatorname{Req}_{D}\right)\right) \backslash S n p\right. \\
&\left.\cup\left(\operatorname{out}(\bar{C}) \backslash \operatorname{Req} q_{C}\right)\right) \cap \operatorname{Req} \\
& S n p \supseteq\left(\operatorname{out}(C) \cup\left(\operatorname{out}(\bar{D}) \backslash \operatorname{Req} q_{D}\right)\right) \cap(\mathbf{u s e}(\bar{C}) \cup(\operatorname{Req} \backslash S b k)) \\
& \operatorname{Re} q_{D} \supseteq(\operatorname{out}(\bar{D}) \backslash S n p) \cap(\operatorname{use}(\bar{C}) \cup(\operatorname{Req} \backslash S b k)) \\
& R e q_{C} \supseteq(\operatorname{out}(\bar{C}) \backslash S b k) \cap \operatorname{Req} .
\end{aligned}
$$

Notice the cycles in these inequations. If we add a variable into $S n p$, we may be allowed to remove it from $R e q_{D}$, and vice versa: as we said, there is no unique best solution. Let's look for the minimal solutions, i.e. the solutions to the equations we obtain by replacing the " $\supseteq$ " sign by a simple "=".

Solving for the Unknown Sets. Manipulation of these equations is tedious and error-prone. Therefore, we have been using a symbolic computation system (e.g. Maple [8]). Basically, we have inlined the equation of, say, Snp into the other equations, and so on until we obtained fixed point equations with a single unknown $X$ of the form

$$
X=A \cup(X \cap B),
$$

whose solutions are of the form " $A$ plus some subset of $B$ ". The solutions are expressed in terms of the following sets:

$$
\begin{aligned}
& S n p_{0}=\operatorname{out}(C) \cap(\operatorname{use}(\bar{C}) \cup(\operatorname{Req} \backslash \text { out }(\bar{C}))) \\
& O p t_{1}=\operatorname{Req} \cap \operatorname{out}(\bar{C}) \cap \text { use }(\bar{C}) \\
& O p t_{2}=\operatorname{Req} \cap \text { out }(\bar{C}) \backslash \text { use }(\bar{C}) \\
& O p t_{3}=\operatorname{out}(\bar{D}) \cap(\text { use }(\bar{C}) \cup \operatorname{Req}) \backslash \operatorname{out}(C) .
\end{aligned}
$$


For each partition of $O p t_{1}$ in two sets $O p t_{1}^{+}$and $O p t_{1}^{-}$, and similarly for $O p t_{2}$ and $\mathrm{Opt}_{3}$, the following is a minimal solution of our problem:

$$
\begin{aligned}
& S b k=O p t_{1}^{+} \cup O p t_{2}^{+} \\
& S n p=S n p_{0} \cup O p t_{2}^{-} \cup O p t_{3}^{+} \\
& R e q_{D}= \\
& R e q_{C}=O p t_{1}^{-} \cup O p t_{2}^{-} . O p t_{3}^{-}
\end{aligned}
$$

Any quadruplet of sets $\left(S b k, S n p, R e q_{D}, R e q_{C}\right)$ that preserves the derivatives (compared to the no-checkpoint code) is equal or larger than one of these minimal solutions. Notice that $O p t_{1} \subseteq S n p_{0}$, and $S n p_{0}, O p t_{2}$, and $O p t_{3}$ are disjoint.

\section{Discussion and Experimental Results}

The final decision for sets $S b k, S n p, R e q_{D}$, and $R e q_{C}$ depends on each particular context. No strategy is systematically best. We looked at two options.

We examined first the option that was implemented until recently in our AD tool TAPENADE 7]. We call it "eager snapshots". This option stores enough variables in the snapshots to reduce the sets $R e q_{D}$ and $R e q_{C}$ as much as possible, therefore reducing the number of subsequent PUSH/POP in $\bar{D}$ and $\bar{C}$. Equations (7) show that we can even make these sets empty, but experiments showed that making $R e q_{D}$ empty can cost too much memory space in some cases.

As always, the problem behind this is undecidability of array indexing: since we can't always tell whether two array indexes designate the same element or not, the "eager snapshot" strategy may end up storing an entire array whereas only one array element was actually concerned.

Therefore "eager snapshot" chooses $O p t_{1}^{-}$and $O p t_{2}^{-}$empty but

$$
\begin{aligned}
O p t_{3}^{+} & =\operatorname{out}(\bar{D}) \cap(\operatorname{use}(\bar{C}) \backslash \operatorname{Req}) \backslash \operatorname{out}(C) \\
O p t_{3}^{-} & =\operatorname{out}(\bar{D}) \cap \operatorname{Req} \backslash \operatorname{out}(C)
\end{aligned}
$$

which gives:

$$
\begin{aligned}
S b k= & \operatorname{Req} \cap \operatorname{out}(\bar{C}) \\
S n p= & (\operatorname{out}(C) \cap(\mathbf{u s e}(\bar{C}) \cup \operatorname{Req} \backslash \operatorname{out}(\bar{C}))) \cup \\
& (\operatorname{out}(\bar{D}) \cap \operatorname{use}(\bar{C}) \backslash \operatorname{Req} \backslash \operatorname{out}(C)) \\
R e q_{D}= & \operatorname{out}(\bar{D}) \cap \operatorname{Req} \backslash \operatorname{out}(C) \\
R e q_{C}= & \emptyset .
\end{aligned}
$$

Notice that intersection between $S b k$ and $S n p$ is nonempty, and requires a special stack mechanism to avoid duplicate storage space.

We examined another option that is to keep the snapshot as small as possible, therefore leaving most of the storage work to the TBR mechanism inside $\bar{D}$ and $\bar{C}$. We call it "lazy snapshots", and it is now the default strategy in TAPENADE. Underlying is the idea that the TBR mechanism is efficient on arrays because when an array element is overwritten by a statement, only this element is saved. 
Therefore, "lazy snapshot" chooses all $O p t_{1}^{+}, O p t_{2}^{+}$, and $O p t_{3}^{+}$empty, yielding:

$$
\begin{aligned}
& S b k=\emptyset \\
& S n p=\operatorname{out}(C) \cap(R e q \cup \operatorname{use}(\bar{C})) \\
& R e q_{D}=\operatorname{out}(\bar{D}) \cap(R e q \cup \operatorname{use}(\bar{C})) \backslash \operatorname{out}(C) \\
& R e q_{C}=\operatorname{out}(\bar{C}) \cap R e q .
\end{aligned}
$$

We ran TAPENADE on our validation application suite, for each of the two options. The results are shown in Table 1. We observe that lazy snapshots perform better in general. Actually, we could show the potential advantage of eager snapshots only on a hand-written example, where the checkpointed part $C$ repeatedly overwrites elements of an array in Req, making TBR mechanism more expensive than a global snapshot of the array. On real applications, however, this case is rare and lazy snapshots work better.

Table 1. Comparison of the eager and lazy snapshot approaches on a number of small to large applications

\begin{tabular}{|l|l|r|r|r|r|} 
Code & Domain & Orig. time & Adj. time & Eager (8) & Lazy (9) \\
\hline \hline OPA & oceanography & $110 \mathrm{~s}$ & $780 \mathrm{~s}$ & $480 \mathrm{Mb}$ & $479 \mathrm{Mb}$ \\
\hline STICS & agronomy & $1.8 \mathrm{~s}$ & $35 \mathrm{~s}$ & $229 \mathrm{Mb}$ & $229 \mathrm{Mb}$ \\
\hline UNS2D & CFD & $2.7 \mathrm{~s}$ & $23 \mathrm{~s}$ & $248 \mathrm{Mb}$ & $185 \mathrm{Mb}$ \\
\hline SAIL & agronomy & $5.6 \mathrm{~s}$ & $17 \mathrm{~s}$ & $1.6 \mathrm{Mb}$ & $1.5 \mathrm{Mb}$ \\
\hline THYC & thermodynamics & $2.7 \mathrm{~s}$ & $12 \mathrm{~s}$ & $33.7 \mathrm{Mb}$ & $18.3 \mathrm{Mb}$ \\
\hline LIDAR & optics & $4.3 \mathrm{~s}$ & $10 \mathrm{~s}$ & $14.6 \mathrm{Mb}$ & $14.6 \mathrm{Mb}$ \\
\hline CURVE & shape optim & $0.7 \mathrm{~s}$ & $2.7 \mathrm{~s}$ & $1.44 \mathrm{Mb}$ & $0.59 \mathrm{Mb}$ \\
\hline SONIC & CFD & $0.03 \mathrm{~s}$ & $0.2 \mathrm{~s}$ & $3.55 \mathrm{Mb}$ & $2.02 \mathrm{Mb}$ \\
\hline Contrived example & $0.02 \mathrm{~s}$ & $0.1 \mathrm{~s}$ & $8.20 \mathrm{Mb}$ & $11.72 \mathrm{Mb}$ \\
\hline
\end{tabular}

Whatever the option chosen, equations (7) naturally capture all interactions between successive snapshots. For example, if several successive snapshots all use an array A, and only the last snapshot overwrites A, it is well known that A must be saved only in the last snapshot. However, when an AD tool does not rely on a formalization of checkpointing such as the one we introduce here, it may very well happen that A is stored by all the snapshots.

\section{Conclusion}

We have formalized the checkpointing technique in the context of reverse AD by program transformation. Checkpointing relies on saving a number of variables and several options are available regarding which variables are saved and when. Using our formalization and with the help of a symbolic computation system, we found that no option is strictly better than all others and we could specify all the 
possible optimal options. This gives us safer and more reliable implementation in $\mathrm{AD}$ tools.

We selected two possible optimal options and implemented them in the AD tool TAPENADE. Experience shows that the option called "lazy snapshots" performs better on most cases.

However, we believe that for reverse AD of a given application code, the option chosen need not be identical for all checkpoints. This formal description of all the possible options allows us to look for the best option for each individual checkpoint, based on static properties at this particular code location. In this regard, we used symbolic computation again and came up with a very pleasant property: for a given checkpoint, whatever the optimal option chosen for the snapshot, the out set of this piece of code turns out to be always the same:

$$
\operatorname{out}(\overline{C ; D})=(\operatorname{out}(\bar{C}) \cup((\operatorname{out}(\bar{D}) \cup \operatorname{out}(C)) \backslash \mathbf{u s e}(\bar{C}))) \backslash \operatorname{Req} .
$$

If checkpoints are nested, this out set is what influences possible enclosing checkpoints. Therefore the choice of the optimal option is local to each checkpoint.

One of the current big challenges of reverse AD is to find the best possible placement of nested checkpoints. This was found [3] for one simple model case. For arbitrary programs, our formulas show that the segmentation of a code into the subsections $U, C$, and $D$ has substantial impact on the memory usage, and they can help finding good such segmentations.

\section{References}

1. A. Aho, R. Sethi, and J. Ullman. Compilers: Principles, Techniques and Tools. Addison-Wesley, 1986.

2. C. Faure and U. Naumann. Minimizing the tape size. In G. Corliss, C. Faure, A. Griewank, L. Hascoët, and U. Naumann, editors, Automatic Differentiation of Algorithms: From Simulation to Optimization, Computer and Information Science, chapter 34, pages 293-298. Springer, New York, NY, 2001.

3. Andreas Griewank. Achieving logarithmic growth of temporal and spatial complexity in reverse automatic differentiation. Optimization Methods and Software, 1:35-54, 1992.

4. Andreas Griewank. Evaluating Derivatives: Principles and Techniques of Algorithmic Differentiation. Number 19 in Frontiers in Appl. Math. SIAM, Philadelphia, PA, 2000.

5. L. Hascoët and M. Araya-Polo. The adjoint data-flow analyses: Formalization, properties, and applications. In H. M. Bücker, G. Corliss, P. Hovland, U. Naumann, and B. Norris, editors, Automatic Differentiation: Applications, Theory, and Tools, Lecture Notes in Computational Science and Engineering. Springer, 2005.

6. L. Hascoët, U. Naumann, and V. Pascual. "to be recorded" analysis in reverse-mode automatic differentiation. Future Generation Computer Systems, 21(8), 2004.

7. L. Hascoët and V Pascual. Tapenade 2.1 user's guide. Technical report 0300, INRIA, 2004. http://www.inria.fr/rrrt/rt-0300.html.

8. Darren Redfern. The Maple handbook, Maple V, release 4. Springer, 1996. 